\title{
Compartments and the Topography of Leg Afferent Projections in Drosophila
}

\author{
R. K. Murphey, ${ }^{1}$ D. R. Possidente, ${ }^{1}$ P. Vandervorst, ${ }^{2}$ and A. Ghysen ${ }^{2}$ \\ ${ }^{1}$ Neurobiology Research Center, Department of Biology, State University of New York, Albany, New York 12222, and \\ 2Laboratory of Genetics, Universite Libre de Bruxelles, 1640 Rhode St Genese, Belgium
}

\begin{abstract}
The legs of Drosophila are covered with mechanosensory bristles, innervated by sensory neurons that project to the CNS in a very orderly manner. We examined this afferent projection by staining the sensory neurons associated with identified bristles in wild-type, engrailed and scute flies. We observe that anterior neurons project to an anterior region of the ventral neuropil, while posterior neurons project to a more posterior region. We rule out that this difference depends on the compartment of origin of the receptors. Our results also argue against explanations based on other factors that might correlate to anterior/posterior position: peripheral organization of the leg nerve, competitive interactions, or differences in times of birth. We suggest that position itself is the primary determinant of this projection.
\end{abstract}

The sensory neurons of insects form an orderly afferent projection from their peripherally located somata to the ganglion of their segment of origin. A number of mechanisms have been implicated in the spatial ordering of the axonal terminals in the CNS, such as the position of the cell body (Ghysen, 1980; Murphey et al., 1980), its time of appearance (Palka et al., 1986), its developmental history (Anderson and Bacon, 1979), its segmental determination (Ghysen et al., 1983), and competitive interactions with other neurons (Murphey, 1986). We investigated the weight of these factors in the case of a large and morphologically near-homogeneous population of sense organs: the mechanosensory bristles on the legs of Drosophila. Presumably, the postsynaptic cells play an important role in this process because their dendrites are the scaffold upon which the sensory neurons elaborate their arbors. However, the role of the postsynaptic cells will not be discussed here. Modality is also a very important correlate of afferent projection (Murphey et al., 1985), but by concentrating only on the singly innervated mechanoreceptor bristles, we have eliminated this issue from consideration.

The neurons underlying the mechanosensory bristles of a leg all project to a large nucleus within the corresponding ganglion, called the leg neuromere by Power (1948) (Murphey et al., in

\footnotetext{
Received Jan. 23, 1989; revised Feb. 27, 1989; accepted Mar. 2, 1989.

We would like to thank Dr. U. Rudolfsky, who provided access to the irradiation facility of the New York State Department of Health and Ms. Kathleen Whitlock for her expertise with the SEM and for providing some of the examples of cobaltstained afferents. R.K.M. is supported by a Jacob Javits Neuroscience Investigator Award (NS 15571) from the NIH. A.G. is Chercheur Qualifie of the Fonds National de la Recherche Scientifique (Belgium).

Correspondence should be addressed to Dr. R. K. Murphey, Neuroscience and Behavior Program, University of Massachusetts, Morrill Hall, Amherst, MA 01003. Copyright (C) 1989 Society for Neuroscience $0270-6474 / 89 / 093209-09 \$ 02.00 / 0$
}

press). They terminate in different regions of the neuromere according to the position of the bristle they innervate: anterior bristles project to the anterior side of the neuromere, while posterior bristles give rise to a more posterior projection (Teugels and Ghysen, 1983). A difference also exists between anterior and posterior cells in the epidermis; a clonal boundary divides the leg into an anterior and a posterior compartment (Steiner, 1976). This subdivision into 2 compartments (Garcia-Bellido et al., 1973, 1979) results from the differential expression of the gene engrailed (en), which is expressed in posterior but not in anterior epidermal cells (Morata and Lawrence, 1975; Kornberg. 1981; Morata et al., 1983). Since the sensory neurons of insects are closely related to the epidermal cells (Lawrence, 1966), it might be that posterior neurons are similarly distinguished from anterior neurons by the en gene, and the choice of the site of axonal projection depends on this difference.

The idea that compartment identity might control the development of sensory neurons (Lawrence, 1978) is not new. Vandervorst and Ghysen (1980) demonstrated a strong correlation between the compartment to which a neuron belongs and the behavior it elicits, consistent with the idea that compartment identity might control axonal arborization and ultimately synaptogenesis. Palka and colleagues (1981), on the other hand, have shown that sensory neurons growing in the wing can cross compartment boundaries, suggesting that compartments have no major effect on axonogenesis in the periphery.

In order to determine whether compartment identity is involved in the control of the axonal projection of leg afferents, one needs to examine the projection of sensory neurons one by one, with special reference to cells near the compartment boundary. If the behavior of a sensory neuron is not strictly correlated with the compartment it originates from, then the former cannot be determined exclusively by the latter.

In this paper, we determine the afferent projection of individual leg bristles in Drosophila. We find that the mechanosensory neurons can be assigned to 2 categories based on their location in the leg and on their axon trajectories in the ganglion. In general, axons from anterior neurons arborize in the anterior region of the leg neuromere, while posterior neurons arborize in the posterior region, as previously suggested by Teugels and Ghysen (1983). However, the line that separates neurons with an anterior trajectory from neurons with a posterior trajectory does not coincide with the compartment boundary, suggesting that compartments do not control this aspect of afferent organization. Alternative explanations, such as differential affinity of anterior and posterior neurons for anterior and posterior pioneer neurons or competition between anterior and posterior 


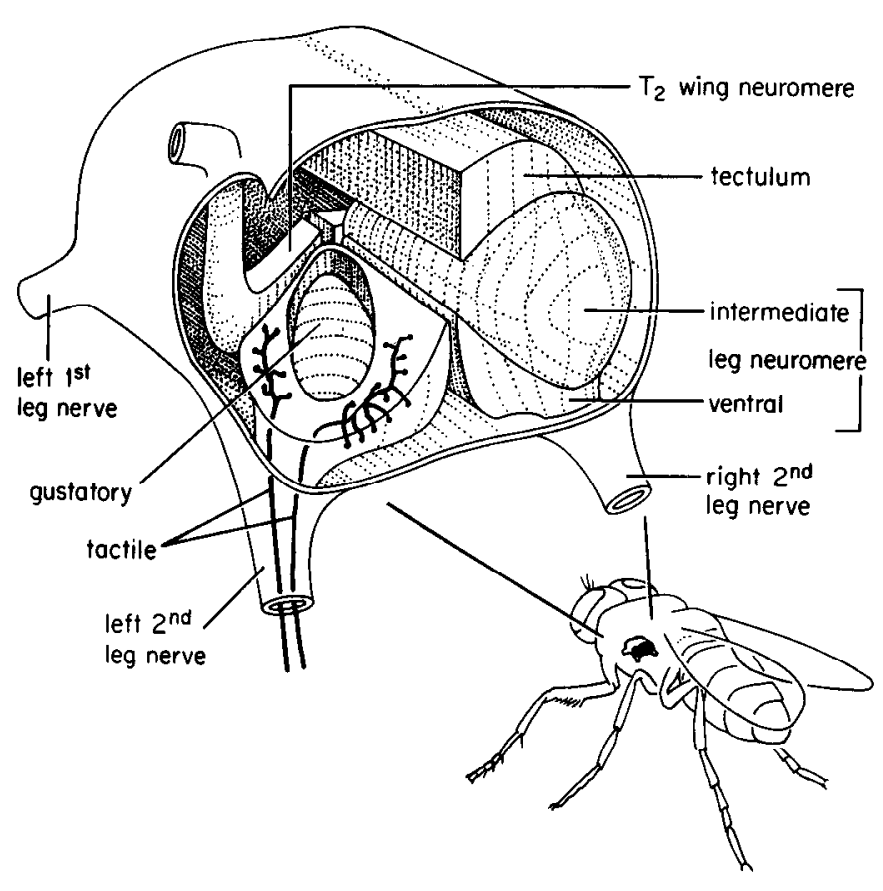

Figure 1. Diagrammatic summary of the organization of the mesothoracic neuromere of Drosophila. The neuromere is viewed from the left rear. The main regions of neuropil are shown on the right half of the ganglion. The left hemiganglion shows a cutaway view of the ventral neuropil. This ventral neuropil consists of an inner region receiving the gustatory afferents and the tactile afferents arborize in a ring around the gustatory neuropil (Details are provided in Possidente and Murphey, 1989; and Murphey et al., in press).

axons, are not supported by our results. We are left with the suggestion that the crucial factor that organizes this projection is the position of the neuron around the leg circumference.

\section{Materials and Methods}

Fly strains. Two wild-type strains were used in these experiments: Cobalt staining was carried out on the Dover strain obtained from D. Mohler of the University of Iowa; HRP staining was done on the Canton S wild type. The en mutations were provided by T. Kornberg of UCSF. The Minute (3) strain was obtained from A. Shearn at Johns Hopkins University. The $s c^{10.1}$ mutation and the unstable ring $\mathrm{X} \operatorname{In}(1) w^{v C}$ were from Ed Lewis of the California Institute of Technology. Stocks were kept at temperatures ranging from 21 to $25^{\circ} \mathrm{C}$.

Staining. Individual neurons were stained with cobalt by breaking the hair in half with forceps and slipping a broken pipette filled with 100 or $200 \mathrm{~mm}$ cobalt over the stub of the hair. The pipette was held in place with dental wax, and the animal was kept in a humid chamber at $5^{\circ} \mathrm{C}$ for $12-20 \mathrm{hr}$. The thoracic nervous system was then exposed by removing the legs and ventral thoracic cuticle, and the specimen was processed using the Timms method of whole-mount intensification (Johnson and Murphey, 1985). The success rate for this method was approximately $50 \%$ (ranging from 20 to $75 \%$ in different batches). The wild-type results reported here are based on 74 successful preparations summarized in Figure 2.

In a second method, the neurons innervating single leg bristles were stained with HRP by pulling the bristle in the presence of the HRP solution as described by Ghysen (1980). The CNS was then processed as described previously (Ghysen, 1978) and examined as a whole-mount, and the legs were examined to determine the identity of the bristles that had been removed. In a preliminary analysis we pulled out 2-4 adjacent bristles; this resulted in 2-4 labeled axons projecting to the CNS. For the 2 rows of bristles anterior to the compartment boundary, where adjacent bristles may follow different trajectories, we pulled individual bristles. The wild-type results presented here and summarized in Figure 2 are based on 69 successful preparations, 36 involving several bristles in the same row and 33 backfills of individual bristles.
Scanning electron microscopy. Specimens used for SEM were airdried, mounted on studs with silver paste, and sputter-coated with a gold-palladium mixture. They were viewed on an International Scientific Instruments Super MINI-SEM and photographed with Polaroid Type 55 positive/negative film.

Clonal analysis. Clonal analysis of the mesothoracic distal femur was done by the Minute technique (Morata and Ripoll, 1975; Steiner, 1976; Held, 1979; Lawrence et al., 1979). Clones of yellow, non-Minute tissue were generated on both Minute(1) $o^{S p}$ and Minute(3)i $i^{5 s}$ backgrounds. Two crosses were made as diagrammed below (females are on the left, males on the right):

$$
\begin{gathered}
M(1) o^{s p} / F M \sigma \times y \\
y / y \times y ; D p(1 ; 3) s c^{J 4}, y^{+} s c^{14} M(3) i^{5 s} / T M 1 M e^{I} m w h
\end{gathered}
$$

The complete genotype of the yellow' stock that we happened to use was $y ; b w ; e ; c i e y^{R}$. Minute $(1) o^{S_{p}}$ was introduced through the female because it is male lethal. $D p(1 ; 3) s c^{14}, y^{+} s c^{14} M(3) i^{5 s}$ must be introduced through the male (Held, 1979).

Eggs were collected from these crosses at 5-24 and 24-48 hr after egg laying and irradiated with 500 and 1000 rad, respectively, with an Isomedix Gammator Model $38-1$ containing ${ }^{137} \mathrm{Cs}$ to obtain clones respecting the anterior-posterior compartment boundary in the leg. Lines of clonal restriction indicating anterior-posterior compartment boundaries can be detected in legs by irradiating as early as $3 \mathrm{hr}$ after egg laying (Steiner, 1976).

Non-Minute progeny eclosed about $2 \mathrm{~d}$ earlier than Minute and were easily culled because of their dominant genetic markers, Bar or Moire. Female Minute(1) $o^{S P}$ and all Minute( 3 ) $i^{55}$ progeny were screened for yellow, non-Minute clones. All progeny were heterozygous for $b w, e, c i$, and $e y^{R}$.

About $50 \%$ of the Minute progeny showed some yellow tissue, 10 $20 \%$ had a clonc on a $\mathrm{lg}$, and lcss than $1 \%$ could be used to establish the anterior-posterior compartment boundary between bristles 15 and 16 on the mesothoracic distal femur.

Leg clones were diagrammed from live animals; these diagrams were then checked after preserving the legs as follows. Animals with clones were heated to $80^{\circ} \mathrm{C}$ for $10-20 \mathrm{~min}$ in $10 \% \mathrm{KOH}$ and dehydrated in ethanol; femurs were then detached and mounted on slides in Canada balsam. Femurs were placed in the groove between 2 coverslips and covered by a third so they could be rolled from side to side for observation.

en crosses. Severely transformed legs can be obtained by making transheterozygotes of some lethal $e n$ alleles and $\mathrm{en}^{l}$. These trans-heterozygotes are viable, but their legs are so deformed that they must be untangled from the pupal case at eclosion (T. Kornberg, personal communication). The cross we used is diagramed below:

$$
\begin{aligned}
& \text { stw cn en }{ }^{I} / C y O \times c n e n^{C 2 L} / S M 5 \\
& \downarrow \\
& \text { stw } \mathrm{cn} \mathrm{en}^{I / c n} \mathrm{en}^{\mathrm{C2L}}
\end{aligned}
$$

(parents)

Scute mosaics. Males that carry the scute $e^{r-l}$ mutation emerge as weak uncoordinated adults that are unable to walk, feed, or mate. Therefore, we generated mosaic flies by crossing heterozygous females, $\mathrm{sc}^{10-1 /} \mathrm{y} \mathrm{Hw}$ with males carrying the unstable ring $\mathrm{X}$ chromosome, $\operatorname{In}(1) w^{\nu \mathrm{C}}$. This has the disadvantage that, in addition to the semilethality of $\operatorname{In}(1) w^{v C}$, the frequency of the ring $X$ loss drops from $30-40 \%$ when the chromosome is provided by the mother to $<1 \%$ when provided by the father.

\section{Results}

\section{Leg receptors in Drosophila}

Three types of hair-like sensilla can be found on the legs of Drosophila: singly innervated tactile bristles, multiply innervated gustatory bristles, and singly innervated hairs found in clusters near leg joints that are called "hair plates" (Dethier, 1976; Possidente and Murphey, 1989; Murphey et al., in press). Here, we focus on the tactile bristles. There are approximately 400 tactile bristles arranged in rows running parallel to the long axis of the leg. Many of these bristles can be identified in every specimen because of their unique external structure, their unique position or a combination of attributes (Hollingsworth, 1964; Hannah-Alava, 1958; Steiner, 1976; Held, 1979). 

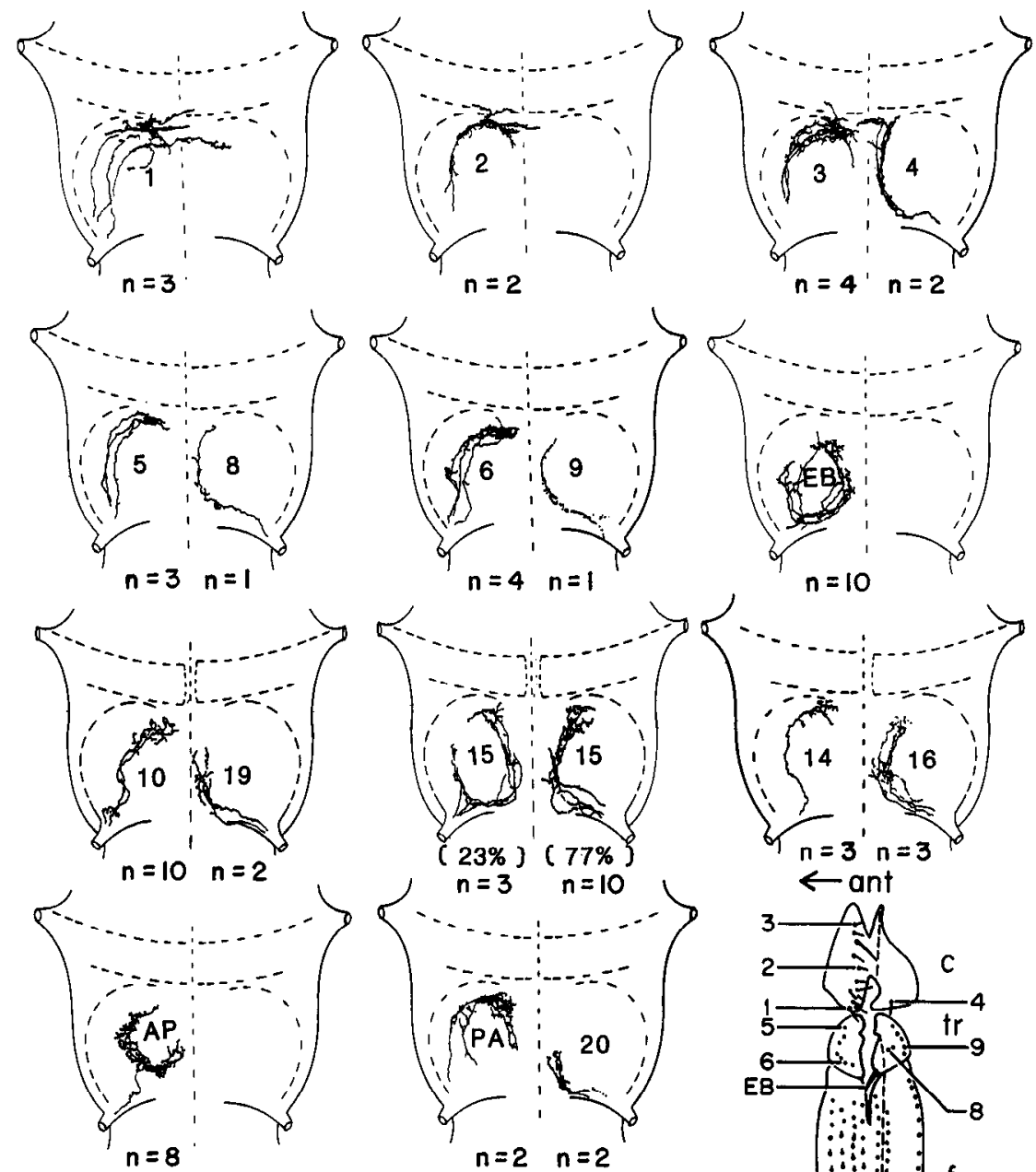

A

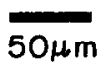

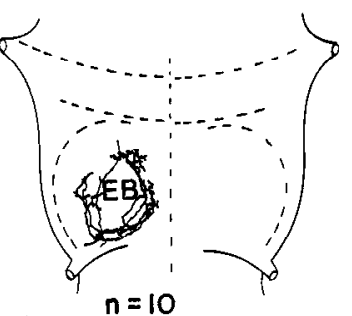

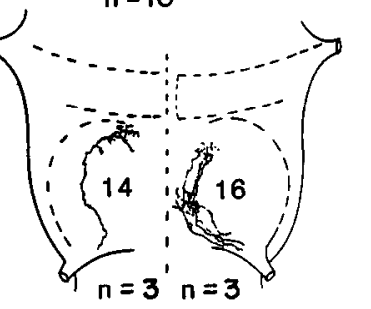

$\leftarrow$ ant

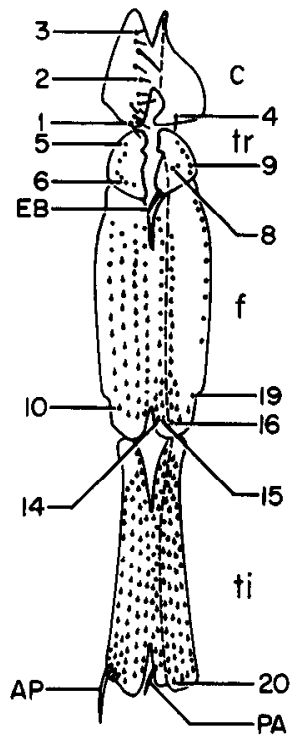

Figure 2. The afferent projection from identified bristles on the mesothoracic leg. $A$, The axonal arborizations of the bristles labeled in $B$. Neurons belonging to the anterior compartment are shown in the left half of the ganglia, neurons in the posterior compartment are shown in the right half of the ganglia (with the exception of neuron 15). This figure is based on 74 successful preparations in which individual neurons were filled using thc cobalt method. Notc that all of the neurons derived from the posterior compartment have axon trajectories and arborizations located posterio-medially. The neurons from the anterior compartment are more variable; note especially the edge bristle of the trochanter $(\mathrm{EB})$ and the apical bristle of the tibia (AP) whose arbors are of intermediate type. The number of examples for each identified neuron in our data set is shown below each example. Most panels contain 2-3 cells to provide some indication of the variability and reliability. When the samplc size is large a maximum of 3 cells are shown. The trajectory and arborization of each cell studied, except neuron 15 , had very high reliability. Neuron 15 is of special interest because it exhibits 2 distinct morphologies (each type is shown separately with the respective proportions in the population indicated). $B$, The map produced by Steiner (1976) showing the location of bristles and the compartment boundaries of the mesothoracic leg. The dashed line represents the dorsal compartment boundary. The receptors are identified by numbers unless they have previously been given names, in such cases the previous name is retained. Anterior is to the left and proximal is at the top. $c$, coxa, $t r$, trochanter, $f$, femur, $t i$, tibia.

\section{Topography of the leg afferent projection}

The organization of the mesothoracic neuromere is summarized in Figure 1. Each sensory neuron on the leg projects an axon to the neuromere associated with that segment. The ventral twothirds of the ganglion receives afferent input from the leg sensory neurons and is known as the "leg neuromere." The neuron associated with each tactile bristle projects an axon through the main leg nerve to the ventral region of the leg neuromere.

The axonal projections from tactile receptors at a variety of locations on the mesothoracic leg are illustrated in Figure 2. Each neuron exhibits an axonal arborization characteristic for that particular neuron. Most neurons exhibit a very consistent morphology, as illustraled in Figure 2, where identified neurons have been superimposed on a single ganglion to illustrate the conservation from animal to animal, as well as the extent of the variability. For example, neuron 1 arborizes anteriomedially in all 3 of the axons shown, but each varies slightly in the extent to which it crosses the midline. In cases where we have many examples such as neuron $10(n=10)$ or the AP neuron $(n=8)$, the structure of the axonal arborization associated with the identified hair is illustrated with a few examples, but all neurons in the sample are similar to the type shown. In other words there is a great deal of consistency in the projections, just as we have come to expect of identified neurons in insects.

The neurons that innervate most leg bristles establish one of 2 basic projections, one that we call "anterior" and one that we call "posterior" (Figs. 1, 2). Neurons 5 and 8 illustrate this feature (Fig. 2): The axon of neuron 5 follows an anterolateral trajectory and that of neuron 8 a posteriomedial trajectory. In 


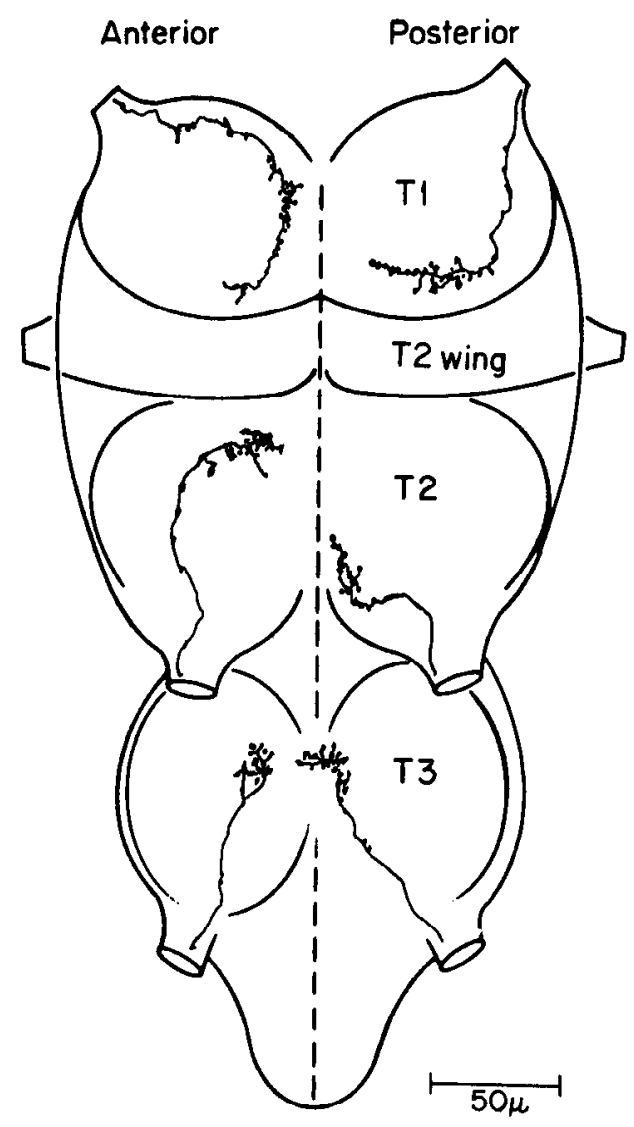

Figure 3. Overview of the adult thoracic and abdominal nervous system illustrating the axonal arborizations of leg sensory neurons in each of the thoracic segments. An anteriorly (left) and a posteriorly (right) located neuron is shown for each segment. Note the shift in axon trajectories that is correlated with the site of entry of the main leg nerves in each segment.

preparations where the leg nerve is clear or in those with more than one stained axon, the nerve appears to contain 2 main bundles projecting to these 2 regions. Axons do cross from one bundle to another at the entrance to the CNS, and thus the axonal trajectory within the ganglion is not obligatorily tied to the bundling in the nerve.

This dichotomous characterization relies heavily on the axon trajectory. However, if the locations of the varicosities or branch points in the arbor are considered, then a more gradual variation is observed, and there appears to be an orderly map of the leg surface in the leg neuromere. Evidence for a correlation with location is provided by the location of axonal branches within the ganglion. As one examines neurons at successive positions around the leg, the axonal arborizations are found at different locations around the leg neuromere. For example, neurons 10 , $14,15,16$, and 19 are located around the distal end of the femur, and their arbors are located at different positions around the neuromere. Thus, although the axonal trajectories can be described as "anterior" or "posterior," the arborizations vary more gradually. In addition, some neurons exhibit axonal arborizations that defy classification as anterior or posterior. For example, neurons 15, AP and EB, have components of both anterior and posterior arborizations (Figs. 2 and 4). In Drosophila our resolution is limited by the small size of the CNS. However, the similarity to the projection patterns observed in the leg of the cricket (Johnson and Murphey, 1985) and moth
(Kent and Levine, 1988), where the larger size makes it easier to establish the topographic order, suggests that the projection pattern represents a "map" of the leg surface.

This pattern of arborization is conserved in the pro- and metathoracic neuromere ganglia. However, in the prothorax the whole pattern is rotated anteriorly to accommodate the anterior entry of the prothoracic leg nerve, and in the metathorax it is rotated more posteriorly (Figs. 3, 4).

\section{Compartments and axon topography}

The boundary dividing the leg into 2 compartments, one anterior and one posterior, has been localized with respect to the tactile bristle rows (Fig. $2 B$; see Steiner, 1976). In order to assess the relation between the afferent projection of a sensory neuron and the compartment of origin of the bristle it innervates, we stained individual bristle neurons on the different segments of the mesothoracic leg (Fig. 2) and also surveyed the femoral bristles of all 3 legs (Fig. 5). In general, neurons that innervate bristles in the anterior compartment have anterior projections, while those from the posterior compartment exhibit posterior projections, as documented earlier by Teugels and Ghysen (1983). However, Figures 2 and 5 show that the change of axon trajectory, from anterior to posterior, does not coincide with the published compartment boundary (Steiner, 1976). Specifically, neurons just anterior to the compartment boundary often have posterior axon trajectories and arborize like their posterior neighbors (e.g., Fig. 2, neuron 15; Fig. 5, double arrowheads).

Since this lack of coincidence is crucial in assessing the role of compartments in the topography of the axonal projections, we examined more closcly the 10 bristles that are located at the distal end of the femur, encompassing all but the ventral rim of the femur (Fig. 2B). Neurons 16-19 are located in the posterior compartment, and each sends an axon to the posteriormedial region of neuropil. Neurons 10-14 are located in the anterior compartment, and their axons terminate anteriormedially. One axon is not correlated with its compartment of origin: neuron 15 is located in the anterior compartment according to Steiner's map (1976) but exhibits a posterior morphology most of the time (10 of 13) and an intermediate morphology in the remaining cases ( 3 of 13). It is noteworthy that this bristle is located just posterior to the dorsal rim of the leg and faces posteriorly. Thus, the axon trajectory of this bristle, and of the others at this circumferential location (Fig. 5), appears to be correlated with its location but not its compartment of origin.

Other examples of neurons that do not fit the anterior-posterior rule can be seen in Figure 2. The axon from the large bristle on the trochanter called the edge bristle (EB), which is derived from the anterior compartment and located near the dorsal compartment border, has both anterior and posterior branches, with the majority of the arbor located posteriomedially. The axon from the apical bristle (AP) of the tibia, which is derived from the anterior compartment and located near the ventral compartment border, has a similar shape and does not fit easily into an anterior-posterior classification.

\section{Compartment of origin of bristle 15}

One possible explanation for the discrepancy between compartment of origin and afferent projection is that individual bristles along the border may originate from either compartment, as has been demonstrated in the tarsus (Lawrence et al., 1979). For example, bristle 15 might be formed by anterior cells 


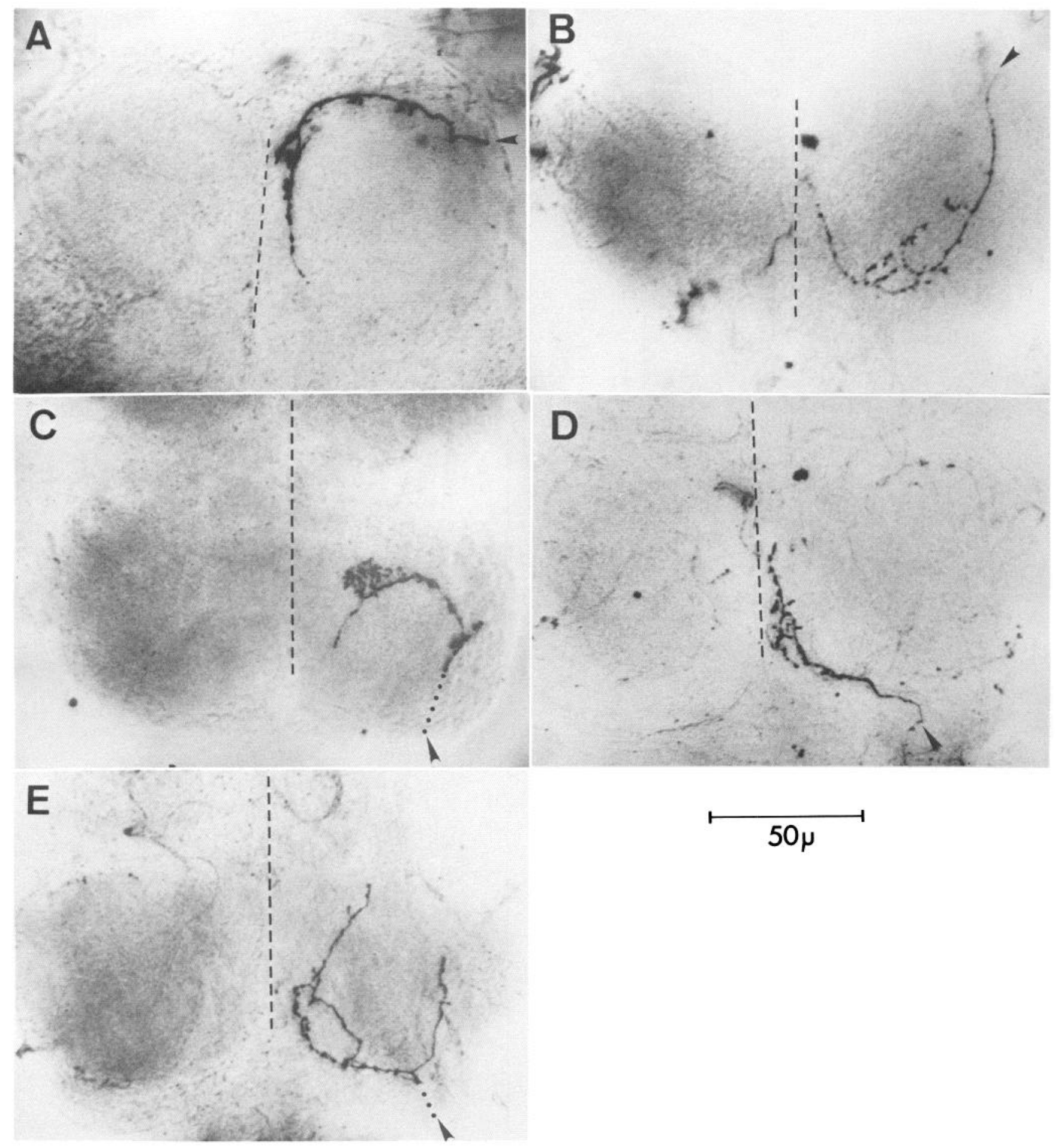

Figure 4. Examples of the axonal arborizations of selected tactile afferents. $A$, Neuron located on the anterior coxa of the prothoracic leg. $B$, Axonal arborization of a neuron located on the posterior coxa of the prothoracic leg. $C$, Apical neuron of the mesothoracic leg. $D$, Neuron 19 from the mesothoracic leg. $E$, Axon associated with the edge bristle (EB) of the mesothoracic trochanter. Each photo shows both leg neuromeres for that segment. As diagramed in Figure 2, the midline is dotted and anterior is at the top. The arrowheads indicate where the axon enters the leg neuromere. The specimens were examined with the aid of an image analysis system described by Tieman et al., 1986.

in some flies and by posterior cells in others. We determined the compartment of origin of this bristle by standard clonal analysis. We induced yellow, non-Minute clones on a Minute background and then searched for clones affecting one or the other compartments at the distal end of the femur (see Materials and Methods). Whenever receptor 15 was marked with the yel- low phenotype, it was derived from a clone belonging to the anterior compartment $(n=5)$. Posteriorly derived clones that included receptor 16 never $(n=5)$ included receptor 15; thus, the compartment boundary always ran between bristles 15 and 16 as first demonstrated by Steiner (1976). As a control, we stained axons 14-19 in the Minute stocks and confirmed that 


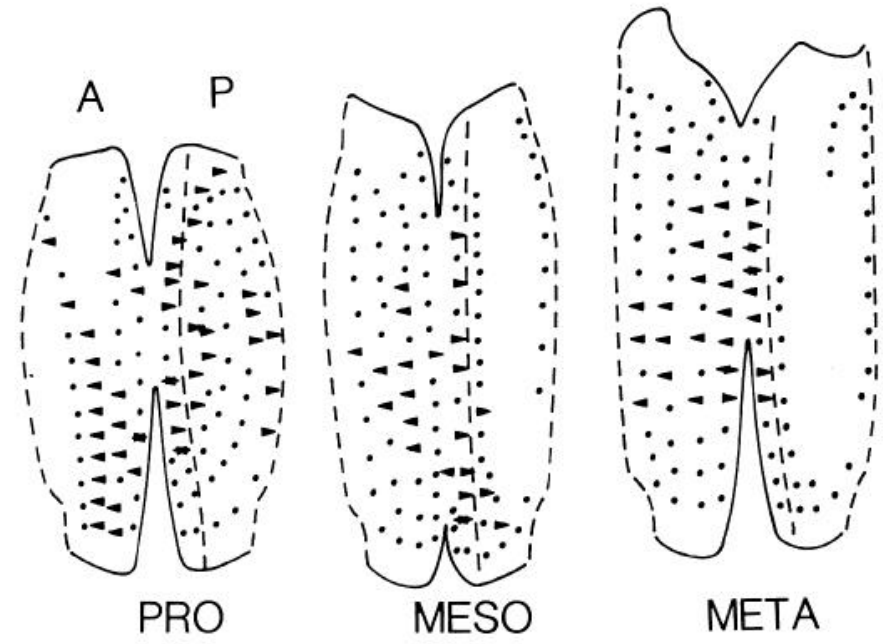

Figure 5. The position of the axon trajectory for sensory neurons on the 3 legs. The diagram shows the positions of the bristles (dots) and dorsal compartment boundaries (dashed lines) of pro-, meso- and metathoracic femurs, according to Steiner (1976). The anterior compartment is to the left. The results are based on 69 successful HRP backfills, 33 of which involve single bristles and the other 36 a few adjacent bristles in a given row. For each bristle that was filled, an arrowhead shows the type of trajectory that was observed (anterior trajectory: arrowhead to the left, posterior trajectory: arrowhead to the right). Note that near the dorsal compartment border, the same identified sensory neuron can have one or the other trajectory in different specimens (double-headed arrows). This variability was never observed for the anterior bristles further from the compartment border, nor for any posterior bristle.

they arborize in the same pattern as wild type (not shown). Based on this evidence, as well as the previously published location of the compartment boundary (Steiner, 1976; Held, 1979), we conclude that receptor 15 is always derived from the anterior compartment. The fact that the axonal arbor of neuron 15 is variable but the compartment of origin is constant implies that axonal termination is independent of compartment identity.

\section{The afferent projection in en flies}

In order to provide an independent test of the influence of compartments on the organization of the leg projection, we examined flies mutant for the en gene. In such mutants, the posterior part of each leg is to some extent "anteriorized" (Brasted, 1941; Tokunaga, 1961). In some combinations of mutant alleles, such as $e n^{1} / e n^{2}$, the posterior to anterior transformation is quite extreme; the transformation often leads to the formation of a posterior duplicate of the large, anterior bristle called the preapical (Fig. 6, arrows; also labeled PA in Fig. 2).

The normal preapical neuron exhibits an anterior trajectory $(n=3$, Fig. 7). When we stain the neuron innervating the duplicate preapical bristle, we observe that the axon trajectory is posterior and the axonal arborization is located in a posterior region appropriate to normal neurons at this location $(n=6)$. In fact, in en animals the afferent projection was completely normal ( $n=13$ successful fills of other leg afferents). These results mean that axonal projections are correlated with their position but not their compartment identity. Thus, we have separated the external appearance of the receptor, which depends on en expression, from the behavior of the neuron, which does not.

\section{The afferent projection in scute mosaics}

We considered the possibility that fibers might compete for targets (Murphey, 1986) or pathways (Gao and Macagno, 1987). For example, if anterior cells were in some way at an advantage over their posterior neighbors, they might take the prime synaptic space and relegate their neighbors to less attractive space. In order to test this possibility, we deleted large numbers of receptors in either the anterior or the posterior compartment and analyzed the projection of the remaining neurons. This was achieved by taking advantage of the mutation scute $e^{10-1}$, which prevents the formation of sensory neurons in the differentiating leg (Jan et al., 1985). This mutation is located on the X chromosome, making it possible to generate $\mathrm{XX} / \mathrm{XO}$ gynanders where the male parts are void of bristles. The boundary between nor-
Figure 6. Scanning electron micrographs of normal and en legs. $A$, Distal end of the tibia of a normal leg. Arrow indicates the preapical bristle. $B$, Tibia of an $e n^{\prime} / e n^{2}$ animal. Arrows indicate preapical bristles. The tarsal segments are transformed into a knob by this genotype.
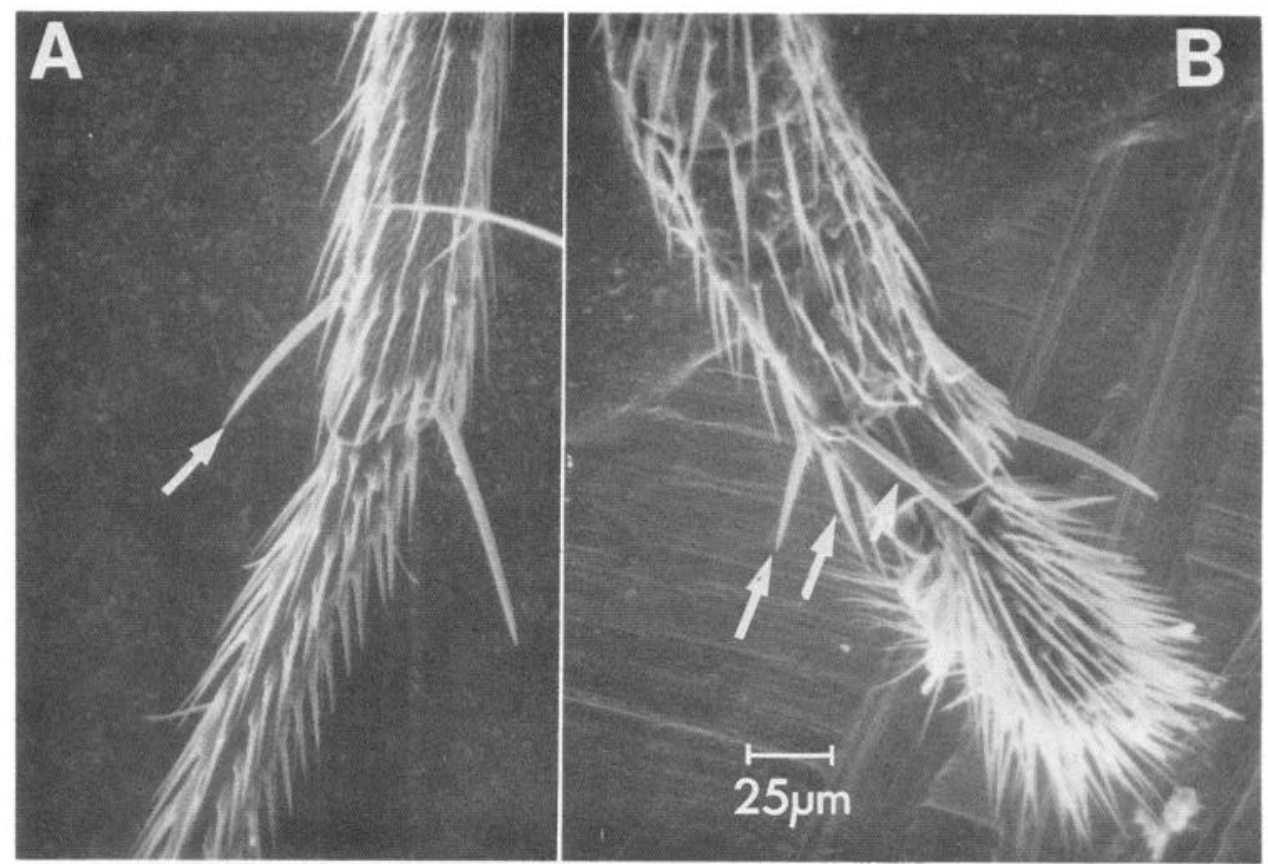


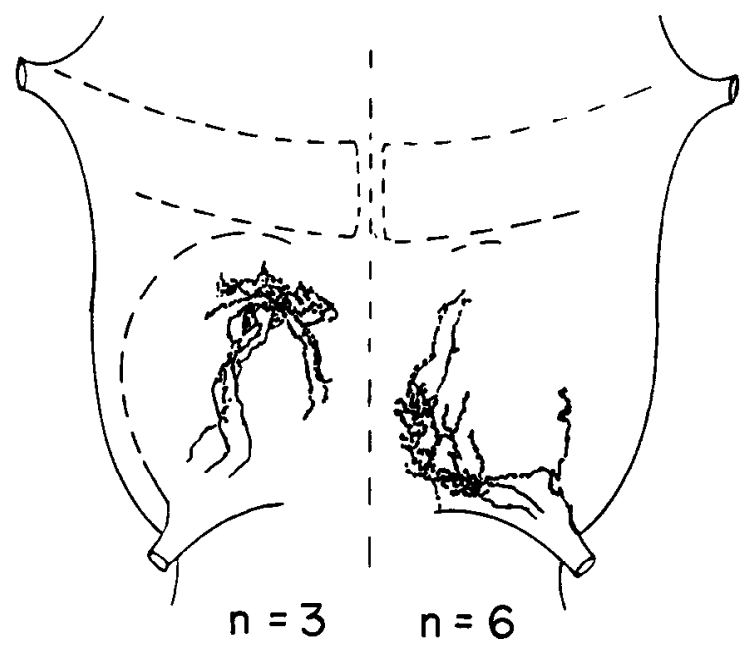

$50 \mu \mathrm{m}$

Figure 7. The axonal arborizations of the sensory neurons associated with preapical bristles in $\mathrm{en}$ flies. On the left 3 axonal arbors of anterior preapicals are superimposed on 1 ganglion outline. On the right side of the same outline 3 arbors from duplicated preapical bristles in en flies are shown. Note that the duplicate preapical arborizes in the same manner as all other sensory neurons from the posterior compartment; it has a posterior axon trajectory and a posteriomedial axonal arborization.

mal and scute territory may occasionally run through a leg, resulting in a mosaic appendage. In all cases, the neurons that were backfilled established a projection appropriate to their location, irrespective of the presence or absence of the other leg bristles. One remarkable case is illustrated in Figure 8 , where all 3 legs of a single gynander were mosaic. The open symbols show the bristles that were pulled out in order to stain the underlying sensory neuron; the resulting projection was typical of posterior bristles in all 3 cases. We conclude from these experiments that the segregation of axon terminals to the anterior and posterior target areas is not under the control of competitive interactions.

\section{Discussion}

\section{The axonal projections from leg sensory neurons}

A striking characteristic of the leg projection in flies is that most individual neurons establish either an anterior or a posterior axon trajectory in the leg neuromere. However, examination of the arborizations, as opposed to the axon trajectories, suggests that the target area for leg bristle afferents is not strictly divided into 2 nonoverlapping regions but rather forms a "map" of the leg surface. The resolution of this map is relatively low because of the small size of the fly. However, the data are sufficient to show strong similarities with larger insects. In the cricket Acheta and the moth Manduca, a highly ordered afferent projection cxists. Like topographic maps in the vertebrate nervous system, the relationship between sensory surface and central projection is often contorted, but there can be no doubt that there is a topographic relationship between the sensory surface (the leg) and the afferent projection (Johnson and Murphey, 1985; Kent and Levine, 1988). The similarity between the leg afferent projections seen in flies and other insects supports the idea that the projection is a highly ordered one, and it can legitimately be called a topographic projection.
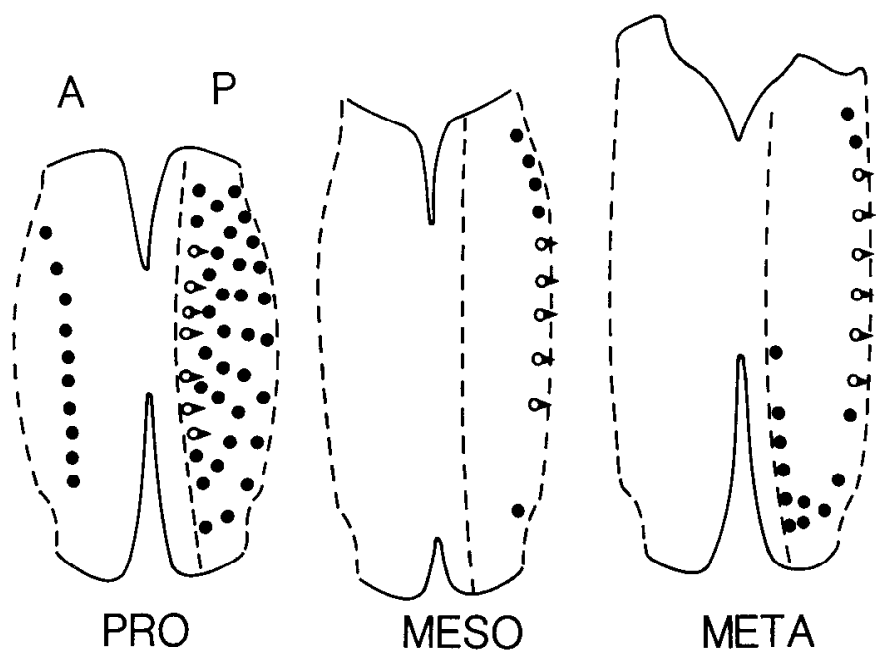

Figure 8. The axon trajectories of sensory neurons of legs mosaic for the scute $e^{10-l}$ mutation. In this genotype many of the receptors are missing. The conventions and symbols are as in Figure 5. Circles represent the bristles that were present; open circles mark the bristles that were backfilled with HRP. For each leg, 5-7 bristles were pulled. In all 3 cases the projection was purely posterior, showing that even in the absence of all bristles on the anterior femur the posterior neurons continuc to cxhibit the appropriatc postcrior axon trajcetory.

\section{Compartments do not control the organization of the leg projection}

Our results show that the projections of sensory neurons are correlated with but not obligatorily tied to their compartment of origin. All axons from the posterior compartment have posterior trajectories, and most axons in the anterior compartment have anterior trajectories. However, receptors in the row just anterior to the compartment boundary usually project as their posterior neighbors; they have posterior axon trajectories and posteriormedial arborizations. For example, bristle 15, which is always derived from the anterior compartment and is located next to the compartment boundary, usually ( $77 \%$ of the time) arborizes like its posterior neighbors (Fig. 2). This has the effect of dividing the leg projection along topographic rather than compartment lines, the anterior face projecting anteriorly in the $\mathrm{CNS}$ and the posterior face, including some cells of anterior compartmental origin, projecting more posteriorly. The fact that compartment identity and axon projection can be uncoupled in wild-type flies suggests that the segregation of anterior and posterior afferents is not under compartmental control.

This conclusion is strengthened by the fact that the afferent projection in en flies is essentially normal. More specifically, posterior bristles in en legs may exhibit the external morphology characteristic of anterior bristles and yet retain a projection typical of normal posterior bristles. Thus, the data on wild-type and en flies provides strong evidence against a role for compartments in the organization of the leg afferent projection.

\section{Alternative mechanisms}

Role of pioneer fibers. A number of other mechanisms appear to be eliminated by our data. One possible explanation for the rough correspondence between trajectory and compartmental origin is based on the existence in the differentiating leg of 2 pioneer bundles, one anterior and one posterior (Jan et al., 1985). In the leg, neurons choosing to follow the nearest pioneer bundle would naturally run in 2 tracts, one anterior and one posterior 
(Bate, 1976; Bentley and Keshishian, 1982). We have not directly tested this possibility; however, this explanation appears unlikely for the following reasons. First, we have observed that the axons of anterior and posterior bristles do, in fact, travel as 2 distinct bundles in the leg nerve; however, this peripheral organization breaks down when the axons enter the CNS, as some fibers clearly leave the tract they follow peripherally and shift to the other side of the neuromere where they arborize. Second, we observed that the leg projection is always normal in $e n^{1 / e n^{2}}$ flies, though the posterior pioneer bundle is absent in more than $90 \%$ of the differentiating legs in early pupae of this genotype (Jan et al., 1985). Because this mutant combination has a low viability, we cannot rule out that the few adults that hatch might come from exceptional larvae in which the 2 pioneers were present. Thus, none of the available evidence supports the view that the presence of 2 pioneer bundles in the differentiating leg determines the behavior of sensory axons in the CNS, although we cannot completely rule out the possibility.

Time of birth of leg neurons. In the wing of the fly, where the distal sensilla give rise to a dual projection in the CNS, the choice between the 2 alternative pathways depends on the time of birth of the neurons (Palka et al., 1986). A similar explanation is excluded in the case of the leg projection since the first anterior and posterior neurons differentiate and begin axonogenesis at the same time (Jan et al., 1985).

Competition. We considered the possibility that some mechanism would split the afferent axon bundle in 2 halves when it enters the CNS. Competition for pathways might accomplish this. For example, in the leech it has been demonstrated that axons can compete for pathways; there appears to be a hierarchy of choices such that when the preferred neuron is not occupying a particular pathway a homolog in another ganglion will do so (Gao and Macagno, 1987). Our observations on scute mosaics argue strongly against this hypothesis, as they show that the remaining neurons will project as expected from their position on the leg, even if most of the neighbors projecting on the other pathway have been deleted.

Competitive interactions within the axonal arborizalions have been revealed recently in the cricket cercal sensory system (Murphey, 1986; Shepherd and Murphey, 1986). A priori a similar mechanism might be responsible for the subdivision of the leg projection in 2 components. The results for the scute mutation appear to eliminate this possibility as well. Genetically removing large numbers of receptors and assessing the afferent projection of some of the remaining receptors had no obvious effect: the patterns of projections were always as expected from the position of the stained neurons. This conclusion must be viewed with caution until the arbors have been studied quantitatively at both light microscopic and ultrastructural levels. A similar result had been obtained in the case of the notum bristles, which also retain a normal projection even if most of the surrounding bristles are removed (Ghysen, 1980). Thus, there is no indication so far that competitive interactions play a role in the organization of afferent projections in the fly, contrary to what has been found in the cricket (Murphey, 1986; Shepherd and Murphey, 1986).

\section{Does positional information organize the leg afferent projection?}

In many developing systems, cells that occupy defined positions will display a defined behavior or undergo a defined transformation, be it in terms of mitotic activity, determination, mi- gration, or differentiation. This has led to the double hypothesis that the position of a cell at the time of its terminal division can specify its differentiation and that cells can measure their position by reference to some system of "positional information" (Wolpert, 1969; French et al., 1976). For example, in the case of sense organs, epidermal tissue transplanted to an ectopic site can be induced to produce receptors that it would not normally make, proving that something about the new position can determine the ultimate fate of the ectopic tissue (Walthall and Murphey, 1984, 1986).

We have shown that the leg projection in flies is correlated with the peripheral position of the receptor. Further, we found no evidence to support other factors such as compartment of origin or competitive advantage. It has been proposed in the fly (Ghysen, 1980) and cricket (Murphey et al., 1980; Walthall and Murphey, 1986) that the topography of the afferent projection depends directly on the position of the neuron (or of the ectodermal cell that will generate the neuron and the other elements of the receptor). In the cricket, strong evidence in support of the idea has been developed using surgery to alter the positions of the neurons (Walthall and Murphey, 1986; Kamper and Murphey, 1987). The present results suggest that this is a reasonable hypothesis for the leg afferent projection in flies as well.

\section{References}

Anderson, H., and J. Bacon (1979) Developmental determination of neuronal projection patterns from wind-sensitive hairs in the locust, Schistocerca gregaria. Dev. Biol. 72: 364-373.

Bate, C. M. (1976) Pioneer neurones in an insect embryo. Nature 260: $54-56$.

Bentley, D. N., and H. Keshishian (1982) Pathfinding by peripheral pioneer neurons in grasshoppers. Science 218: 1082-1088.

Brasted, A. (1941) An analysis of the expression of the mutant "engrailed" in Drosophila melanogaster. Genetics 26: 347-373.

Dethier, V. G. (1976) The Hungry Fly, Harvard U. P., London.

French, V. I., P. J. Bryant, and S. V. Bryant (1976) Pattern regulation in epimorphic fields. Science 193: 969-981.

Gao, W.-Q., and E. R. Macagno (1987) Neurons in the leech depend upon the existence of neighboring homologues. I. The HA cells. J. Neurobiol. 18: 43-60.

Garcia-Bellido, A., P. Ripoll, and G. Morata (1973) Developmental compartmentalisation of the wing disc of Drosophila. Nature [New Biol.] 245: 251-253.

Garcia-Bellido, A., P. A. Lawrence, and G. Morata (1979) Compartments in animal development. Sci. Am. 241: 102-110.

Ghysen, A. (1978) Sensory neurones recognise defined pathways in Drosophila central nervous system. Nature 274: 869-872.

Ghysen, A. (1980) The projection of sensory neurons in the central nervous system of Drosophila: Choice of the appropriale pathway. Dev. Biol. 78: 521-541.

Ghysen, A., R. Janson, and P. Santamaria (1983) Segmental determination of sensory neurons in Drosophila. Dev. Biol. 99: 7-26.

Hannah-Alava, A. (1958) Morphology and chaetotaxy of the legs of Drosophila melanogaster. J. Morphol. 103: 281-310.

Held, L. I. (1979) A high-resolution morphogenetic map of the secondleg basitarsus in Drosophila melanogaster. Wilhelm Roux's Arch. 187: $129-150$.

Hollingsworth, M. J. (1964) Sex-combs of intersexes and the arrangement of the chaetae on the legs of Drosophila. J. Morphol. 115:3552.

Jan, Y. N., A. Ghysen, I. Christoph, S. Barbel, and L. Y. Jan (1985) Formation of neuronal pathways in the imaginal discs of Drosophila melanogaster. J. Neurosci. 5: 2453-2464.

Johnson, S. E., and R. K. Murphey (1985) The afferent projection of mesothoracic bristle hairs in the cricket, Acheta domesticus. J. Comp. Physiol. A 156: 369-379.

Kamper, G., and R. K. Murphey (1987) Synapse formation by sensory neurons after cross species transplantation in crickets: The role of positional information. Dev. Biol. 122: 492-502.

Kent, K. S., and R. B. Levine (1988) Neural control of leg movements 
in a metamorphic insect: Sensory and motor elements of the larval thoracic legs in Manduca sexta. J. Comp. Neurol. 271: 559-576.

Kornberg, T. (1981) Engrailed: A gene controlling compartment and segment formation in Drosophila. Proc. Natl. Acad. Sci. USA 78: 1095-1099.

Lawrence, P. A. (1966) Development and determination of hairs and bristles in the milkweed bug Oncopeltus fasciatus. J. Cell. Sci. 1:475498.

Lawrence, P. A. (1978) Compartments and the insect nervous system. Zoology 6: 157-160.

Lawrence, P. A., G. Struhl, and G. Morata (1979) Bristle patterns and compartment boundaries in the tarsi of Drosophila. J. Embryol. Exp. Morph. 51: 195-208.

Morata, G., and P. Lawrence (1975) Control of compartment development by the engrailed gene in Drosophila. Nature 255: 214-217.

Morata, G., and P. Ripoll (1975) Minutes: mutants of Drosophila autonomously affecting cell division rate. Dev. Biol. 42: 211-221.

Morata, G., T. Kornberg, and P. A. Lawrence (1983) The phenotype of engrailed mutations in the antenna of Drosophila. Dev. Biol. 99: $27-33$.

Murphey, R. K. (1986) Competition and the dynamics of axon arbor growth in the cricket. J. Comp. Neurol. 251: 100-110.

Murphey, R. K., A. Jacklet, and L. Schuster (1980) A topographic map of sensory cell terminal arborizations in the cricket CNS: Correlation with birthday and position in a sensory array. J. Comp. Neurol. 191: 53-64.

Murphey, R. K., J. P. Bacon, and S. E. Johnson (1985) Ectopic neurons and the organization of insect sensory systems. J. Comp. Physiol. A 156: 381-389.

Murphey, R. K., D. Possidente, G. Pollak, and D. J. Merritt (in press) Modality specific axonal projections in the CNS of the flies Phormia and Drosophila. J. Comp. Neurol.

Palka, J., M. Schubiger, and H. S. Hart (1981) The path of axons in Drosophila wings in relation to compartment boundaries. Nature 294: 447-449.
Palka, J., M. A. Malone, R. L. Ellison, and D. J. Wigston (1986) Central projections of identified Drosophila sensory neurons in relation to their time of development. J. Neurosci. 6: 1822-1830.

Possidente, D., and R. K. Murphey (1989) Genetic control of sexually dimorphic axon morphology in Drosophila sensory neurons. Dev. Biol. 132: 448-457.

Power, M. E. (1948) The thoracico-abdominal nervous system of an adult insect, Drosophila melanogaster. J. Comp. Neurol. 88:347-409.

Shepherd, D., and R. K. Murphey (1986) Competition regulates the efficacy of an identified synapse in crickets. J. Neurosci. 6: 3152 3160 .

Steiner, E. (1976) Establishment of compartments in the developing leg imaginal discs of Drosophila melanogaster. Wilhelm Roux's Arch. 180: 9-30.

Teugels, E., and A. Ghysen (1983) Two mechanisms for the establishment of sensory projections in Drosophila. Prog. Brain Res. 58: 305-312.

Tieman, D. G., R. K. Murphey, J. T. Schmidt, and S. B. Tieman (1986) A computer-assisted video technique for preparing high resolution pictures and stereograms from thick specimens. J. Neurosci. Meth. 17: $231-245$.

Tokunaga, C. (1961) The differentiation of a secondary sex comb under the influence of the gene engrailed in Drosophila melanogaster. Genetics 46: 157-176.

Vandervorst, P., and A. Ghysen (1980) Genetic control of sensory connections in Drosophila. Nature 286: 65-67.

Walthall, W. W., and R. K. Murphey (1984) Rules for neural development revealed by chimaeric sensory systems in crickets. Nature 311: 57-59.

Walthall, W. W., and R. K. Murphey (1986) Positional informations compartments and the cercal sensory system of crickets. Dev. Biol. 113: 182-200.

Wolpert, L. (1969) Positional information and the spatial pattern of cellular differentiation. J. Theor. Biol. 25: 1-47. 